\title{
Posterior atrophy predicts time to dementia in patients with amyloid-positive mild cognitive impairment
}

Jung-Min Pyun ${ }^{1,2 \dagger}$, Young Ho Park ${ }^{1,2+}$, Hang-Rai Kim ${ }^{3}$, Jeewon Suh ${ }^{1}$, Min Ju Kang ${ }^{1}$, Beom Joon Kim¹, Young Chul Youn ${ }^{5}$, Jae-Won Jang ${ }^{4 *}$, SangYun Kim ${ }^{1,2^{*}}$ (D) and the Alzheimer's Disease Neuroimaging Initiative

\begin{abstract}
Background: In patients with amyloid-positive mild cognitive impairment (MCI), neurodegenerative biomarkers such as medial temporal lobe atrophy (MTA) are useful to predict disease progression to dementia. Although posterior atrophy (PA) is a well-known neurodegenerative biomarker of Alzheimer's disease, little is known about PA as a predictor in patients with amyloid-positive $\mathrm{MCl}$.

Methods: We included 258 patients with amyloid-positive $\mathrm{MCl}$ with at least one follow-up visit, and who had low cerebrospinal fluid (CSF) $\beta$-amyloid ${ }_{1-42}$ concentration. Data were obtained from the Alzheimer's Disease Neuroimaging Initiative study. We assessed PA and MTA on magnetic resonance imaging (MRI) using visual rating scales and retrospectively determined progression to dementia during the follow-up period of up to 3 years (median 24 months). The Cox proportional hazards model was used to analyze hazard ratios (HRs) of PA and MTA for disease progression. Additionally, subjects were divided into four groups according to brain atrophy pattern (no atrophy, MTA only, PA only, both MTA and PA), and HRs for disease progression were compared with the no atrophy reference group. Analyses were conducted with and without adjustment for CSF phosphorylated tau $181 \mathrm{p}$ (p-tau) and baseline demographics.
\end{abstract}

Results: A total of 123 patients (47.7\%) showed MTA and 174 patients (67.4\%) showed PA. Of the total cohort, 139 cases (53.9\%) progressed to dementia. PA and MTA were associated with an increased risk for progression to dementia (HR 2.244, 95\% confidence interval (CI) 1.497-3.364, and HR 1.682, 95\% Cl 1.203-2.352, respectively). In the analysis according to atrophy pattern, HR (95\% Cl) for progression was 2.998 (1.443-6.227) in the MTA only group, 3. 126 (1.666-5.864) in the PA only group, and 3.814 (2.045-7.110) in both MTA and PA group. These results remained significant after adjustment.

Conclusions: In patients with amyloid-positive $\mathrm{MCl}, \mathrm{PA}$ could predict progression to dementia independently of MTA.

Keywords: Posterior atrophy, Biomarkers, Disease progression, Mild cognitive impairment, Alzheimer's disease

\footnotetext{
* Correspondence: light26@kangwon.ac.kr; neurosky@snu.ac.kr

${ }^{\dagger}$ Equal contributors

${ }^{4}$ Department of Neurology, Kangwon National University Hospital, 156,

Baengnyeong, Chuncheon, Kangwon 24341, Republic of Korea

${ }^{1}$ Department of Neurology, Seoul National University Bundang Hospital, 82,

Gumi-ro 173 Beon-gil, Bundang-gu, Seongnam-si, Gyeonggi-do 13620,

Republic of Korea

Full list of author information is available at the end of the article
}

(c) The Author(s). 2017 Open Access This article is distributed under the terms of the Creative Commons Attribution 4.0 International License (http://creativecommons.org/licenses/by/4.0/), which permits unrestricted use, distribution, and reproduction in any medium, provided you give appropriate credit to the original author(s) and the source, provide a link to the Creative Commons license, and indicate if changes were made. The Creative Commons Public Domain Dedication waiver (http://creativecommons.org/publicdomain/zero/1.0/) applies to the data made available in this article, unless otherwise stated. 


\section{Background}

Dyshomeostasis of $\beta$-amyloid $(A \beta)$ is an initiating factor of Alzheimer's disease (AD) pathology [1]. A $\beta$ positivity, defined as $A \beta$ deposition on positron emission tomography (PET) or a low level of cerebrospinal fluid (CSF) $\beta$-amyloid -42 $_{1-4}\left(\mathrm{~A} \beta_{1-42}\right)$, is known to predict clinical progression from mild cognitive impairment (MCI) to dementia [2, 3]. However, using only $A \beta$ positivity for the prediction of disease progression is limited by the fact that $A \beta$ positivity in CSF begins at least 15 years before expected clinical symptom onset [4]. Therefore, neurodegenerative biomarkers that reflect sequential pathologic processes after $A \beta$ positivity could be useful in predicting progression from MCI to dementia in the near future $[5,6]$.

Neurodegeneration is the process of neuronal injury, and can be evaluated by atrophy observed on structural magnetic resonance imaging (MRI), hypometabolism on $\left[{ }^{18} \mathrm{~F}\right]$-fluorodeoxyglucose-PET, positive tau PET, or increased CSF tau [6-8]. Medial temporal lobe atrophy (MTA) on MRI is one of the most well-known neurodegenerative markers [9], and can predict progression from MCI to dementia $[10,11]$. In a study with MCI patients with $\mathrm{A} \beta$ positivity, those with MTA were more likely to progress to dementia, which indicates the predictive value of MTA in amyloid-positive MCI [5].

Recently, an increasing number of studies have suggested that another neurodegenerative marker, posterior atrophy (PA) on MRI, could also predict conversion to dementia in MCI $[11,12]$. In particular, evidence has shown that, whereas MTA is related to low levels of CSF $A \beta_{1-42}$, PA is associated with high levels of CSF total tau (t-tau) and phosphorylated $\mathrm{tau}_{181 \mathrm{p}}$ ( $\mathrm{p}$-tau); this might support a prognostic value of PA in terms of disease progression among patients with amyloid-positive MCI [11]. The current study therefore aimed to assess the predictive value of PA for progression to dementia in amyloid-positive MCI.

\section{Methods \\ Subjects}

Data used in the preparation of this article were obtained from the Alzheimer's Disease Neuroimaging Initiative (ADNI) database (adni.loni.usc.edu). The ADNI was launched in 2003 as a public-private partnership, led by Principal Investigator Michael W. Weiner, MD. The primary goal of ADNI has been to test whether serial MRI, PET, other biological markers, and clinical and neuropsychological assessments can be combined to measure the progression of MCI and early AD. For upto-date information, see www.adni-info.org.

Data used in this study were downloaded from the ADNI database on 25 May, 2017. We included patients with late MCI who had had a baseline MRI scan, amyloid positivity on a CSF study [13], and at least one or more follow-up visits after initial assessment. The primary outcome of this study was progression to dementia during the follow-up period of up to 3 years. A final total of 258 patients, 143 from the ADNI1 cohort and 115 from the ADNI2 cohort, were included in this study.

Diagnosis of late MCI was made according to the presence of objective memory impairment but without meeting the criteria for dementia. Namely, all subjects had a Mini Mental State Examination (MMSE) score of 24 or higher, a global Clinical Dementia Rating (CDR) score of 0.5 , a CDR memory score of 0.5 or higher, and a score indicating impairment on the delayed recall of Story A of the Wechsler Memory Scale-Revised ( $\geq 16$ years of education: $\leq 8 ; 8-15$ years of education: $\leq 4 ; 0-7$ years of education: $\leq 2$ ), which is 1.5 standard deviations below the mean score of cognitively normal subjects.

\section{CSF measurements and cutoffs}

Baseline CSF $A \beta_{1-42}$, t-tau, and $p$-tau were measured centrally using the multiplex xMAP Luminex platform (Luminex Corp, Austin, TX, USA) with Innogenetics (INNO-BIA AlzBio3, Ghent, Belgium; for research useonly reagents) immunoassay kit-based reagents, as described by Shaw and colleagues [13]. Qualification of the analytical performance of CSF samples from ADNI was controlled, showing within-center coefficient of variation $(\% \mathrm{CV})$ 95\% confidence interval $(\mathrm{CI})$ value (mean) of $4.0-6.0 \%$ (5.3\%) for $A \beta_{1-42}, 6.4-6.8 \%$ (6.7\%) for $\mathrm{t}$-tau, and $5.5-18.0 \%$ (10.8\%) for $\mathrm{p}$-tau [14]. Intercenter \%CV 95\% CI ranged from 15.9 to $19.8 \%$ (17.9\%) for $A \beta_{1-42}, 9.6-15.2 \%$ (13.1\%) for $t-t a u$, and $11.3-18.2 \%$ (14.6\%) for p-tau [14]. Amyloid positivity was defined as CSF $\mathrm{A} \beta_{1-42}$ of less than $192 \mathrm{pg} / \mathrm{ml}$ [13].

\section{MRI}

Brain MRI scans were acquired as previously described [15]. Participants in the ADNI1 cohort were scanned using either a $1.5 \mathrm{~T}$ or $3 \mathrm{~T}$ MRI scanner. All subjects in the ADNI2 cohort were scanned using a $3 \mathrm{~T}$ MRI scanner.

\section{MTA scale}

MTA was evaluated using a five-point rating scale developed by Scheltens et al. [16]. According to the height of the hippocampal formation and the width of the choroidal fissure and the temporal horn, atrophy was rated from 0 (no atrophy) to 4 (severe atrophy) (Additional file 1: Table S1). The largest vertical height of hippocampal formation was defined as dentate gyrus, hippocampus proper, subiculum, and parahippocampal gyrus.

\section{PA scale}

PA was assessed using a four-point rating scale developed by Koedam et al. $(0=$ no atrophy; $1=$ mild widening of the 
sulci without evident volume loss of gyri; $2=$ substantial widening of the sulci and volume loss of the gyri; $3=$ severe end-stage atrophy) [17]. The evaluated anatomical regions included the posterior cingulate sulcus, precuneus, parieto-occipital sulcus, and the cortex of the parietal lobes. MRI scans were assessed in the three different orientations by following anatomical landmarks: widening of the posterior cingulate and parieto-occipital sulcus and atrophy of the precuneus in the sagittal orientation, widening of the posterior cingulate sulcus and the sulcal dilatation in the parietal lobes in the axial orientation, and widening of the posterior cingulate sulcus and parietal lobes in the coronal orientation. In case of different rating scores between different MRI planes, the higher score was used for analysis.

\section{Image analysis}

All MRI scans were evaluated by three raters (board-certified neurologists, Young Ho Park, Hang-Rai Kim, Jeewon Suh, with 7, 5, and 4 years of experience in dementia) who were blinded to the clinical information. In case of disagreement, the three raters reviewed the MRI scans together for adjustment.

Intra-rater reliability was assessed by the re-rating of 25 randomly determined MRI scans at a separate sitting, blinded to their own prior rating. Inter-, and intra-rater reliabilities were measured by calculating the intraclass correlation coefficient.

For rating scores of both MTA and PA, scores of the right and left hemispheres for each patient were summed and the mean value was used. These scores were dichotomized as normal (no atrophy) or abnormal (atrophy). For MTA in those younger than 75 years old a rating score of 1.5 or more was considered abnormal, and in those aged 75 years or older a score of 2 or more was considered abnormal [18]. For PA, a score of 1.5 or more was considered abnormal in all patients [17].

For quantitative analysis we used data downloaded from the ADNI database. Regional volumes were measured automatically by the Freesurfer image analysis suite, which is freely available for download (http://surfer.nmr.mgh.harvard.edu/). ADNI1 1.5 T data were run with Freesurfer version 4.3, and ADNI1 $3 \mathrm{~T}$ data and ADNI2 data were run with Freesurfer version 5.1. Each scan was segmented according to an atlas defined by Freesurfer [19]. We compared volumes of the hippocampus, parahippocampal cortex, entorhinal cortex, and fusiform gyrus in temporal regions as well as the superior and inferior parietal cortex, precuneus, supramarginal gyrus, and postcentral gyrus in parietal regions between groups with and without progression to dementia, using the Mann-Whitney test or Student's $t$ test as appropriate.

\section{Statistical analysis}

For comparison of demographic and clinical variables between groups with and without progression to dementia, we used the Pearson chi-squared test, Mann-Whitney test, or Student's $t$ test as appropriate. We assessed the hazard ratio (HR) of MTA, PA, CSF t-tau, p-tau, baseline demographics, and neuropsychological profiles using univariate Cox regression analysis with follow-up time as a time variable and progression to dementia as a status variable. Additionally, we categorized MRI atrophy pattern into the following four groups: no atrophy, MTA only, PA only, and both MTA and PA. The HR of each group for disease progression was calculated using univariate Cox analysis. The proportional assumption was examined by $\log$-log survival plots.

The multivariate Cox analysis was performed to identify independent determinants of disease progression with relevant covariates. The clinically or statistically relevant covariates with a $p$ value $<0.2$ in univariate Cox regression analysis were included. If there was more than one variable that was clinically highly correlated, we included only one of them in the model. Also, according to the atrophy pattern, two different models were implemented. In model 1, HRs of the MTA group and PA group were analyzed with adjustment for clinically or statistically relevant covariates. In model 2, HRs of the four groups according to atrophy pattern (no atrophy, MTA only, PA only, both MTA and PA) were analyzed with adjustment for relevant covariates. Multicollinearity between the covariates was tested by calculating the variance inflation factor [20]. We used SPSS 21 (SPSS Inc., Chicago, Illinois, USA) for multicollinearity and inter- and intra-rater reliabilities analyses, and $\mathrm{R}$ (version 3.3.1; http://www.R-project.org) for the remainder of the analyses. Demographic analysis was performed using wilcox.test, chisq.test, or t.test function, and Cox regression analysis was performed using the Coxph function in $\mathrm{R}$ with survival package version 2.41-3.

\section{Results}

A total of 258 patients participated in the study. The median age of patients was 74.1 years, and 101 (39.1\%) were female. A total of 176 patients $(68.2 \%)$ had at least one APOE \&4 allele. Of the total cohort, 123 patients (47.7\%) showed MTA and 174 patients (67.4\%) showed PA. Dividing patients according to atrophy pattern, we identified 51 patients $(19.8 \%)$ with no atrophy, 33 patients $(12.8 \%)$ with MTA only, 84 patients (32.6\%) with PA only, and 90 patients (34.9\%) with both MTA and PA. During the follow-up period (median 24 months) 139 patients (53.9\%) progressed to dementia, and 119 patients did not. Demographic, cognitive, and biomarker characteristics according to progression to dementia, categorized as stable $\mathrm{MCI}$ and progressive $\mathrm{MCI}$, are summarized in Table 1. Patients 
Table 1 Baseline characteristics of the study sample

\begin{tabular}{|c|c|c|c|c|}
\hline & All $(n=258)$ & Stable MCI $(n=119)$ & Progressive $\mathrm{MCl}(n=139)$ & $p$ value $^{c}$ \\
\hline Age, years & $74.1(69.5-78.5)$ & $74.4(69.1-78.2)$ & $73.6(69.8-78.7)$ & $>0.999$ \\
\hline Female, $n(\%)$ & $101(39.1)$ & $40(33.6)$ & $61(43.9)$ & 0.119 \\
\hline Education, years & $16(14-18)$ & $16(14-18)$ & $16(14-18)$ & 0.469 \\
\hline APOE \&4 carrier, $n$ (\%) & $176(68.2)$ & $78(65.6)$ & $98(70.5)$ & 0.473 \\
\hline \multicolumn{5}{|l|}{ Cognition } \\
\hline MMSE & $27(25-29)$ & $28(26-29)$ & $26(25-28)$ & $<0.001$ \\
\hline CDR SB & $1.5(1.0-2.5)$ & $1.5(1.0-2.0)$ & $2.0(1.0-2.5)$ & $<0.001$ \\
\hline ADAS-cog 11 & $11.8(9.0-15.7)$ & $10.7(7.2-13.2)$ & $13.0(10.8-16.2)$ & $<0.001$ \\
\hline \multicolumn{5}{|l|}{ CSF markers } \\
\hline $\mathrm{A} \beta_{1-42}$, mean $\pm \mathrm{SD}, \mathrm{pg} / \mathrm{mL}$ & $136.1 \pm 25.7$ & $136.7 \pm 27.2$ & $133.7 \pm 25.0$ & 0.345 \\
\hline CSF t-tau, pg/mL ${ }^{a}$ & $104.0(77.0-148.5)$ & $91.0(68.0-133.0)$ & $113.0(84.0-153.0)$ & 0.006 \\
\hline CSF p-tau, pg/mL ${ }^{\mathrm{b}}$ & $41.0(31.0-58.0)$ & $37.0(27.0-51.0)$ & $45.0(36.0-64.0)$ & $<0.001$ \\
\hline \multicolumn{5}{|l|}{ MRI } \\
\hline Tesla, $n(\%)$ & & & & 0.714 \\
\hline 1.5 & $143(55.4)$ & $64(53.8)$ & 79 (56.8) & \\
\hline 3.0 & $115(44.6)$ & $55(46.2)$ & $60(43.2)$ & \\
\hline MTA, $n(\%)$ & $123(47.7)$ & $47(39.5)$ & $76(54.7)$ & 0.021 \\
\hline PA, $n(\%)$ & $174(67.4)$ & $65(54.6)$ & 109 (78.4) & $<0.001$ \\
\hline Atrophy pattern, $n(\%)$ & & & & $<0.001$ \\
\hline No atrophy & $51(19.8)$ & 39 (32.8) & $12(8.6)$ & \\
\hline MTA only & $33(12.8)$ & 15 (12.6) & $18(13.0)$ & \\
\hline PA only & $84(32.6)$ & $33(27.7)$ & $51(36.7)$ & \\
\hline Both MTA and PA & $90(34.9)$ & $32(26.9)$ & $58(41.7)$ & \\
\hline
\end{tabular}

Data are presented as the median (interquartile range) unless otherwise specified

${ }^{a}$ Data for 7 subjects were not available

${ }^{b}$ Data for 1 subject were not available

c Stable $\mathrm{MCl}$ vs. progressive $\mathrm{MCl}$

$A \beta \beta$-amyloid, ADAS-cog Alzheimer's Disease Assessment Scale-cognitive subscale, CDR SB Clinical Dementia Rating Sum of Boxes, CSF cerebrospinal fluid, MCI mild cognitive impairment, MMSE Mini-Mental State Examination, MRI magnetic resonance imaging, MTA medial temporal lobe atrophy, $P A$ posterior atrophy, $p$-tau tau phosphorylated at threonine $181, S D$ standard deviation, $t$-tau total tau

with disease progression to dementia had poorer cognitive performances at baseline, higher levels of CSF $\mathrm{t}$-tau and CSF p-tau, and more frequent MTA and PA compared with those without progression. The interrater reliability for MTA (0.84-0.87) and PA (0.70$0.87)$ was good. The intra-rater reliability for MTA (0.83-0.98) was excellent and for PA (0.73-0.98) was good (Additional file 2: Table S2). The volumes of the hippocampus, entorhinal cortex, fusiform gyrus, superior and inferior parietal cortex, precuneus, and supramarginal gyrus were significantly decreased in progressive MCI as compared to stable MCI (Additional file 3: Table S3).

In the univariate Cox regression analysis, the presence of MTA and PA showed significantly increased HR (95\% CI) of progression to dementia with a value of 1.682 $(1.203-2.352)$ and $2.244 \quad(1.497-3.364)$, respectively (Fig. 1). In the analysis according to MRI atrophy pattern, patients with only MTA, only PA, and both MTA and PA were associated with a higher risk for disease progression compared with those with no atrophy (Table 2). Higher levels of CSF t-tau and CSF p-tau were related with more disease progression. Baseline cognitive performances with lower MMSE scores, higher CDR Sum of Boxes (CDR $\mathrm{SB})$, and higher Alzheimer's Disease Assessment Scalecognitive subscale 11 (ADAS-cog 11) scores were also associated with progression to dementia. The proportional assumption was satisfied for MTA and PA based on loglog survival plots (Additional file 4: Figure S1).

Multivariate Cox analysis included clinically (age, sex) and statistically relevant variables (education duration, APOE \&4 allele, ADAS-cog 11, CDR-SB, and CSF p-tau) (Table 2). Although MMSE and CSF t-tau were statistically relevant and variance inflation factors were less than 1.719 for all variables, indicating a low degree of collinearity, we excluded them from the multivariate Cox analysis because they were clinically highly correlated with ADAS-cog 11 and CSF p-tau, respectively. 


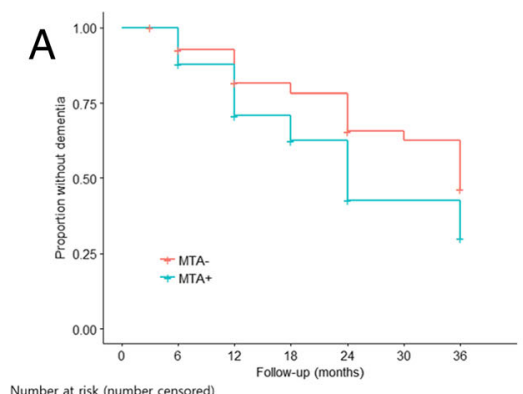

Number at risk (number censored)

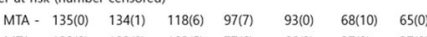

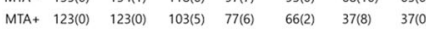

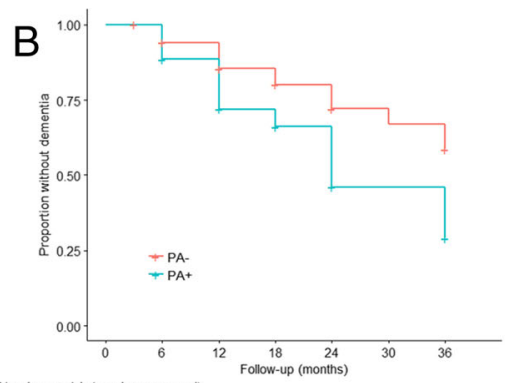

Number at risk (number censored)
$\begin{array}{ccccccc}\mathrm{PA}-84(0) & 83(1) & 76(2) & 65(4) & 60(1) & 43(11) & 40(0)\end{array}$

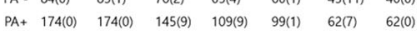

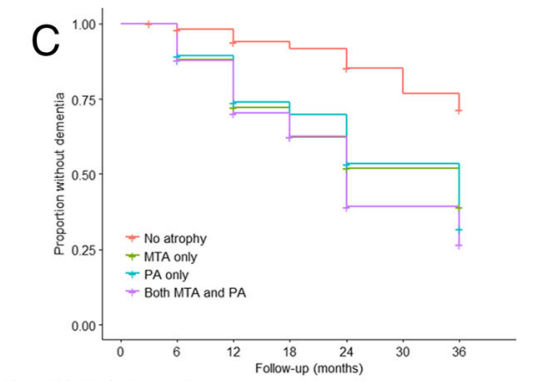

$\begin{array}{cccccccc}\text { Number at risk (number censored) } & & & & & \\ \text { No atrophy } & 51(0) & 50(1) & 48(1) & 43(3) & 42(0) & 31(8) & 28(0)\end{array}$

$\begin{array}{llllllll}\text { No atrophy } & 51(0) & 50(1) & 48(1) & 43(3) & 42(0) & 31(8) & 28(0) \\ \text { MTA only } & 33(0) & 33(0) & 28(1) & 22(1) & 18(1) & 12(3) & 12(0)\end{array}$ $\begin{array}{llllllll}\text { PA only } & 84(0) & 84(0) & 70(5) & 54(4) & 51(0) & 37(2) & 37(0)\end{array}$

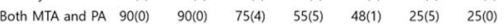

Fig. 1 Cox proportional hazards model for progression to dementia in amyloid-positive mild cognitive impairment patients according to brain atrophy on MRI. PA (a), MTA (b), and atrophy pattern (no atrophy, MTA only, PA only, both MTA and PA) (c). MTA medial temporal lobe atrophy, PA posterior atrophy

Table 2 Univariate and multivariate Cox regression analysis

\begin{tabular}{|c|c|c|c|}
\hline & \multirow{2}{*}{$\begin{array}{l}\text { Univariate } \\
\text { HR (95\% Cl) }\end{array}$} & \multicolumn{2}{|l|}{ Multivariate } \\
\hline & & $\begin{array}{l}\text { Model } 1 \\
\text { HR (95\% Cl) }\end{array}$ & $\begin{array}{l}\text { Model } 2 \\
\text { HR (95\% Cl) }\end{array}$ \\
\hline Age & $0.999(0.976-1.022)$ & $0.996(0.970-1.023)$ & $0.998(0.972-1.025)$ \\
\hline Female & $1.266(0.906-1.770)$ & $1.152(0.797-1.665)$ & $1.162(0.8031-1.682)$ \\
\hline Education & $0.961(0.907-1.019)$ & $0.954(0.900-1.011)$ & $0.961(0.906-1.020)$ \\
\hline$A P O E$ \&4 carrier & $1.290(0.896-1.858)$ & $0.996(0.674-1.470)$ & $1.031(0.700-1.520)$ \\
\hline \multicolumn{4}{|l|}{ Cognition } \\
\hline MMSE & $0.821(0.748-0.901)^{b}$ & $\mathrm{Nl}$ & $\mathrm{Nl}$ \\
\hline CDR SB & $1.593(1.347-1.883)^{\mathrm{b}}$ & $1.526(1.276-1.824)^{b}$ & $1.531(1.279-1.832)^{b}$ \\
\hline ADAS-cog 11 & $1.118(1.080-1.157)^{\mathrm{b}}$ & $1.101(1.061-1.144)^{b}$ & $1.104(1.063-1.147)^{b}$ \\
\hline \multicolumn{4}{|l|}{ CSF markers } \\
\hline CSF t-tau* & $1.003(1.000-1.006)^{\mathrm{a}}$ & $\mathrm{Nl}$ & $\mathrm{Nl}$ \\
\hline CSF p-tau ${ }^{* *}$ & $1.012(1.006-1.019)^{b}$ & $1.006(1.000-1.013)$ & $1.006(1.000-1.013)$ \\
\hline \multicolumn{4}{|l|}{ MRI } \\
\hline MTA & $1.682(1.203-2.352)^{a}$ & $1.424(0.997-2.034)^{a}$ & $\mathrm{Nl}$ \\
\hline PA & $2.244(1.497-3.364)^{b}$ & $1.895(1.239-2.897)^{b}$ & $\mathrm{Nl}$ \\
\hline \multicolumn{4}{|l|}{ Atrophy pattern } \\
\hline No atrophy & Reference & $\mathrm{NI}$ & Reference \\
\hline MTA only & $2.998(1.443-6.227)^{a}$ & $\mathrm{NI}$ & $3.178(1.520-6.645)^{b}$ \\
\hline PA only & $3.126(1.666-5.864)^{b}$ & $\mathrm{NI}$ & $3.209(1.693-6.080)^{b}$ \\
\hline Both MTA and PA & $3.814(2.045-7.110)^{\mathrm{b}}$ & $\mathrm{NI}$ & $3.598(1.909-6.783)^{b}$ \\
\hline
\end{tabular}

* Data for 7 subjects were not available

** Data for 1 subject were not available

a $p<0.05$

${ }^{\mathrm{b}} p<0.001$

Model 1: adjusted for MTA, PA, age, sex, education, APOE $\varepsilon 4$ carrier, ADAS-cog 11, CDR SB, and CSF p-tau

Model 2: adjusted for MRI atrophy pattern (no atrophy, MTA only, PA only, both MTA and PA), age, sex, education, APOE $\varepsilon 4$ carrier, ADAS-cog 11, CDR SB, and

CSF p-tau

ADAS-cog Alzheimer's Disease Assessment Scale-cognitive subscale, CDR SB Clinical Dementia Rating Sum of Boxes, Cl confidence interval, CSF cerebrospinal fluid, $H R$ hazard ratio, MMSE Mini Mental State Examination, MRI magnetic resonance imaging, MTA medial temporal lobe atrophy, NI not included, PA posterior atrophy, p-tau tau phosphorylated at threonine $181, t$-tau total tau 
The adjusted covariates did not alter the significance of the HRs (95\% CI) of MTA (1.424 (0.997-2.034)) or PA (1.895 (1.239-2.897)). Moreover, the HR of groups with only MTA, only PA, and both MTA and PA remained significant. Notably, there was little difference between the HRs of MTA only, PA only, and both MTA and PA. On the contrary, the significant relationships between CSF p-tau and disease progression disappeared after adjustment with other covariates.

\section{Discussion}

The ability to predict progression from MCI to dementia is increasingly important with the prospect of diseasemodifying therapies. This study demonstrated that patients with amyloid-positive MCI with PA or MTA are more likely to progress to dementia. Furthermore, patients with amyloid-positive MCI with PA only (in the absence of MTA) also showed an increased risk for disease progression. This indicates that PA, as well as MTA, is a predictive marker of conversion from amyloid-positive MCI to dementia.

To understand the pathophysiology of $\mathrm{AD}$, considerable effort has been made to identify AD-related focal regions or functional connectivity between regions in the brain [21-24]. According to pathological staging by Braak and Braak, the neurofibrillary changes (tau pathology) start from the medial temporal lobe and spread to neocortical association areas [25]. Many neuroimaging studies have demonstrated these pathological changes, showing low glucose metabolism and cortical atrophy, most prominently in the medial temporal lobe and parietal lobe, including the bilateral precuneus, posterior cingulate cortex, and angular gyrus [21, 24, 26]. MTA within these regions is widely recognized as an imaging marker of $\mathrm{AD}$ [16]. Additionally, the parietal lobe has been highlighted as an area involved in the pathological changes and dysfunction in $\mathrm{AD}$ [27].

The parietal cortex is originally known for its visuospatial and sensorimotor functions [23]. However, the parietal cortex is also involved in other functions, such as episodic memory retrieval [23, 28]; notably, episodic memory retrieval impairment is a typical feature of AD. Functional MRI (fMRI) studies with memory-related tasks have revealed that the parietal cortex, especially the precuneus and superior and inferior parietal lobules, is involved in memory retrieval [29-31]. Furthermore, resting-state fMRI studies have revealed that patients with MCI display lower levels of neuronal activity in the posterior cingulate cortex, precuneus, and inferior parietal lobe (components of default mode network) compared with healthy controls [32, 33]. Concerning cortical connectivity, these regions comprise the "posterior medial network" (PM network), along with the retrosplenial cortex and parahippocampal cortex [34]. The
PM network and anterior temporal network are two largely segregated pathways with different anatomical regions and different memory-guided behaviors, and were proposed by Ranganath and Ritchey [34]. The PM network is involved in episodic memory, spatial navigation, and scene perception. Dominant disruption of this network has been observed in patients with AD compared with healthy participants and patients with other types of dementia [21, 22, 34].

Systematic assessment of PA using a visual rating scale was suggested by Koedam and colleagues [17]. This visual rating scale, used to rate MRI images for PA within the posterior cingulate gyrus, precuneus, and parietal lobe, is simple, easily implemented in clinical settings, and has a good discrimination ability between healthy individuals and patients with $\mathrm{AD}$ [17]. The visual rating scale has been validated using voxel-based morphometry (VBM), with good reliability [35]. Furthermore, in a study of patients with pathologically proven definite $A D$, $30 \%$ of patients showed only PA without MTA, which indicates that PA could be used as an independent imaging marker of AD [36].

From the perspective of cognitive reserve, $\mathrm{MCI}$ patients with both MTA and PA are expected to progress more rapidly to dementia than patients with MTA only or patients with PA only [37]. However, progression rates were not different between patients with both MTA and PA, patients with MTA only, and patients with PA only in our study. Similar results have been reported regarding clinical progression of patients with $\mathrm{AD}[38,39]$. In those studies, which grouped patients with $\mathrm{AD}$ into three subtypes according to cortical atrophy patterns, patients with diffuse atrophy did not show more rapid progression than patients with medial temporal dominant atrophy or parietal dominant atrophy. Rather, patients with parietal dominant atrophy showed a faster progression rate [38, 39]. Although we do not know the reason, MTA and PA might not have additive effects on clinical progression in patients with amyloid-positive MCI.

With respect to VBM analysis in previous studies with patients with $\mathrm{MCI}$, gray matter differences between patients with progressive and stable MCI were assessed using two-sided $t$ tests in early studies [40]. However, the two-sided $t$ tests discard information about varying lengths of follow-up times among patients. To overcome this problem, time-to-event statistical methods were used in VBM analysis of patients with MCI [41]. These studies revealed that the patients with progressive $\mathrm{MCI}$ showed volume loss in the medial temporal lobes as well as the temporoparietal cortex and frontal lobes compared with patients with stable MCI [40-42]. A recent study, which used a VBM survival analysis and assessed the effects of amyloid deposition on progression to 
dementia in patients with MCI, found that the pattern of decreased gray matter volume that was predictive of progression was similar in amyloid-positive and amyloid-negative patients [42]. Although our study demonstrated the usefulness of visually assessed PA for predicting progression to dementia in patients with amyloid-positive $\mathrm{MCI}$, visually assessed PA might also be useful in patients with amyloid-negative MCI. In our previous study of patients with MCI without information about amyloid positivity, visual rating of PA had predictive value for progression to dementia [12].

Notably, our population showed a relatively large proportion of $A P O E \varepsilon 4$ carriers (62\%). This might be due to characteristics of our amyloid-positive population. Other studies with amyloid-positive MCI also reported a large percentage of $A P O E$ \&4 carriers [5, 43, 44]. A considerably higher prevalence of amyloid positivity has been previously reported among $A P O E \& 4$ carriers compared to $A P O E$ \&4 noncarriers [45]. The relationship between $A P O E \varepsilon 4$ and amyloid positivity has been investigated extensively for its important pathological role and contributing risk to $\mathrm{AD}$. $A P O E \varepsilon 4$ is known to increase $A D$ risk by decreasing $A \beta$ clearance and promoting $A \beta$ aggregation [46]. Apolipoprotein E4 has lower affinity to $A \beta$ than apolipoprotein E3, thus showing inefficient removal of $A \beta$ across the blood-brain barrier and increased oligomerization of $\mathrm{A} \beta$ [47-49]. This aspect could support the large proportion of $A P O E \varepsilon 4$ carriers of our amyloid-positive MCI population.

The significant association found between increased CSF p-tau levels and disease progression in our study is consistent with previous studies [50]. However, this significance disappeared in multivariate analysis, contrary to PA or MTA. This could indicate that brain atrophy might correlate more strongly with clinical progression than CSF biomarkers. Several studies have shown similar correlations between MRI, CSF, and cognitive performance [51,52]. One possible explanation for the stronger relationship of brain atrophy with progression (compared to the relationship of CSF biomarkers with progression) is that MRI may be a more stable biomarker for neuronal injury than CSF tau proteins, which can be influenced by diurnal variation and transient brain injury [51, 52]. Otherwise, disease progression with brain atrophy could be affected by factors other than tauopathy, such as aging, traumatic brain injury, toxic factors, and vascular factors [53-56].

There are some limitations to our study that should be noted. First, we defined amyloid positivity by CSF A $\beta_{1-42}$ levels only, and did not include a population with positive amyloid PET. This may have led to a selection bias. However, several studies have shown there to be a good agreement between CSF $A \beta_{1-42}$ levels and amyloid PET, which could minimize the potential bias in our study
$[57,58]$. Second, the visual rating scale may not be precise compared with volumetric quantitative measurements. Although we compared volumetric measures of temporal and parietal regions between stable and progressive MCI, we could not perform voxel- or surfacebased analysis because our sample comprised ADNI1 and ADNI2 cohorts with different magnetic field strength.

\section{Conclusions}

This study showed that PA in amyloid-positive MCI is significantly associated with disease progression to dementia, independent of the presence of MTA. This is indicative of the predictive value of PA for disease progression in patients with amyloid-positive MCI.

\section{Additional files}

Additional file 1: Table S1. Visual rating of medial temporal lobe atrophy. (DOCX $14 \mathrm{~kb}$ )

Additional file 2: Table S2. Inter- and intra-rater reliability. (DOCX 16 kb)

Additional file 3: Table S3. Comparison of volumetric measures of temporal and parietal regions according to disease progression to dementia. (DOCX $19 \mathrm{~kb}$ )

Additional file 4: Figure S1. Log-log survival plots of PA (A) and MTA (B). MTA medial temporal lobe atrophy, PA posterior atrophy. (TIF $83 \mathrm{~kb}$ )

\section{Abbreviations}

A $\beta$ : $\beta$-Amyloid; AD: Alzheimer's disease; ADAS-cog: Alzheimer's Disease Assessment Scale-cognitive subscale; ADNI: Alzheimer's Disease Neuroimaging Initiative; CDR: Clinical Dementia Rating; CDR SB: Clinical Dementia Rating Sum of Boxes; Cl: Confidence interval; CSF: Cerebrospinal fluid; CV: Coefficient of variation; fMRI: Functional magnetic resonance imaging; HR: Hazard ratio; MCl: Mild cognitive impairment; MMSE: Mini Mental State Examination; MRI: Magnetic resonance imaging; MTA: Medial temporal lobe atrophy; PA: Posterior atrophy; PET: Positron emission tomography; PM: Posterior medial; p-tau: Phosphorylated tau 181 p; t-tau: Total tau; VBM: Voxel-based morphometry

\section{Acknowledgements}

Data collection and sharing for this project was funded by the Alzheimer's Disease Neuroimaging Initiative (ADNI) (National Institutes of Health Grant U01 AG024904) and DOD ADNI (Department of Defense award number W81XWH-12-2-0012). ADNI is funded by the National Institute on Aging, the National Institute of Biomedical Imaging and Bioengineering, and through generous contributions from the following: AbbVie, Alzheimer's Association; Alzheimer's Drug Discovery Foundation; Araclon Biotech; BioClinica, Inc.; Biogen; Bristol-Myers Squibb Company; CereSpir, Inc.; Cogstate; Eisai Inc.; Elan Pharmaceuticals, Inc.; Eli Lilly and Company; Eurolmmun; F. Hoffmann-La Roche Ltd. and its affiliated company Genentech, Inc.; Fujirebio; GE Healthcare; IXICO Ltd.; Janssen Alzheimer Immunotherapy Research \& Development, LLC.; Johnson \& Johnson Pharmaceutical Research \& Development LLC.; Lumosity; Lundbeck; Merck \& Co., Inc.; Meso Scale Diagnostics, LLC.; NeuroRx Research; Neurotrack Technologies; Novartis Pharmaceuticals Corporation; Pfizer Inc.; Piramal Imaging; Servier; Takeda Pharmaceutical Company; and Transition Therapeutics. The Canadian Institutes of Health Research is providing funds to support ADNI clinical sites in Canada. Private sector contributions are facilitated by the Foundation for the National Institutes of Health (www.fnih.org). The grantee organization is the Northern California Institute for Research and Education, and the study is coordinated by the Alzheimer's Therapeutic Research Institute at the University of Southern California. ADNI data are disseminated by the Laboratory for Neuro Imaging at the University of Southern California. The Medical Research Collaborating Center of Seoul National University Bundang Hospital contributed to statistical analyses. 
Data used in the preparation of this article were obtained from the Alzheimer's Disease Neuroimaging Initiative (ADNI) database (adni.loni.usc.edu). As such, the investigators within the ADNI contributed to the design and implementation of ADNI and/or provided data but did not participate in analysis or writing of this report. A complete listing of ADNI investigators can be found at: http://adni.loni.usc.edu/wp-content/uploads/ how_to_apply/ADNI_Acknowledgement_List.pdf

\section{Availability of data and materials}

Not applicable.

\section{Authors' contributions}

YHP, JWJ, and SYK designed the study and participated in data analysis and interpretation. YHP, JWJ, and JMP participated in data analysis and interpretation, drafted the manuscript, and revised the manuscript for important intellectual content. JS, HRK, and BJK participated in data analysis. All authors read and approved the final manuscript.

\section{Ethics approval and consent to participate}

The study procedures were approved by the institutional review board of all participating centers (http://adni.loni.usc.edu/wp-content/uploads/ how_to_apply/ADNI_Acknowledgement_List.pdf) and written informed consent was obtained from all participants or authorized representatives.

\section{Competing interests}

The authors declare that they have no competing interests.

\section{Publisher's Note}

Springer Nature remains neutral with regard to jurisdictional claims in published maps and institutional affiliations.

\section{Author details}

'Department of Neurology, Seoul National University Bundang Hospital, 82, Gumi-ro 173 Beon-gil, Bundang-gu, Seongnam-si, Gyeonggi-do 13620, Republic of Korea. ${ }^{2}$ Department of Neurology, Seoul National University College of Medicine, Seoul, Republic of Korea. ${ }^{3}$ Graduate School of Medical Science \& Engineering, KAIST, Daejeon, Republic of Korea. ${ }^{4}$ Department of Neurology, Kangwon National University Hospital, 156, Baengnyeong, Chuncheon, Kangwon 24341, Republic of Korea. ${ }^{5}$ Department of Neurology, Chung-Ang University Hospital, Seoul, Republic of Korea.

Received: 5 September 2017 Accepted: 22 November 2017

Published online: 16 December 2017

\section{References}

1. Selkoe DJ, Hardy J. The amyloid hypothesis of Alzheimer's disease at 25 years. EMBO Mol Med. 2016;8:595-608.

2. Wolk DA, Price JC, Saxton JA, Snitz BE, James JA, Lopez OL, et al. Amyloid imaging in mild cognitive impairment subtypes. Ann Neurol. 2009;65:557-68.

3. Hansson $\mathrm{O}$, Zetterberg $\mathrm{H}$, Buchhave $\mathrm{P}$, Londos $\mathrm{E}$, Blennow $\mathrm{K}$, Minthon L. Association between CSF biomarkers and incipient Alzheimer's disease in patients with mild cognitive impairment: a follow-up study. Lancet Neurol. 2006:5:228-34

4. Bateman RJ, Xiong C, Benzinger TL, Fagan AM, Goate A, Fox NC, et al. Clinical and biomarker changes in dominantly inherited Alzheimer's disease. N Engl J Med. 2012;367:795-804.

5. van Rossum IA, Vos SJ, Burns L, Knol DL, Scheltens P, Soininen H, et al. Injury markers predict time to dementia in subjects with $\mathrm{MCl}$ and amyloid pathology. Neurology. 2012:79:1809-16.

6. Jack Jr CR, Knopman DS, Jagust WJ, Petersen RC, Weiner MW, Aisen PS, et al. Tracking pathophysiological processes in Alzheimer's disease: an updated hypothetical model of dynamic biomarkers. Lancet Neurol. 2013; 12:207-16.

7. McKhann GM, Knopman DS, Chertkow H, Hyman BT, Jack Jr CR, Kawas CH, et al. The diagnosis of dementia due to Alzheimer's disease: recommendations from the National Institute on Aging-Alzheimer's Association workgroups on diagnostic guidelines for Alzheimer's disease. Alzheimers Dement. 2011:7:263-9.

8. Jack Jr CR, Bennett DA, Blennow K, Carrillo MC, Feldman HH, Frisoni GB, et al. A/T/N: an unbiased descriptive classification scheme for Alzheimer disease biomarkers. Neurology. 2016;87:539-47.
9. Scheltens P, van de Pol L. Impact commentaries. Atrophy of medial temporal lobes on MRI in "probable" Alzheimer's disease and normal ageing: diagnostic value and neuropsychological correlates. J Neurol Neurosurg Psychiatry. 2012:83:1038-40.

10. Korf ES, Wahlund LO, Visser PJ, Scheltens P. Medial temporal lobe atrophy on MRI predicts dementia in patients with mild cognitive impairment. Neurology. 2004;63:94-100.

11. Lehmann M, Koedam EL, Barnes J, Bartlett JW, Barkhof F, Wattjes MP, et al. Visual ratings of atrophy in $\mathrm{MCl}$ : prediction of conversion and relationship with CSF biomarkers. Neurobiol Aging. 2013;34:73-82.

12. Kim HR, Park YH, Jang JW, Park SY, Wang MJ, Baek MJ, et al. Visual rating of posterior atrophy as a marker of progression to dementia in mild cognitive impairment patients. J Alzheimers Dis. 2017:55:137-46.

13. Shaw LM, Vanderstichele $H$, Knapik-Czajka M, Clark CM, Aisen PS, Petersen $\mathrm{RC}$, et al. Cerebrospinal fluid biomarker signature in Alzheimer's disease neuroimaging initiative subjects. Ann Neurol. 2009;65:403-13.

14. Shaw LM, Vanderstichele $H$, Knapik-Czaika M, Figurski M, Coart E, Blennow K et al. Qualification of the analytical and clinical performance of CSF biomarker analyses in ADNI. Acta Neuropathol. 2011:121:597-609.

15. Jack Jr CR, Bernstein MA, Fox NC, Thompson P, Alexander G, Harvey D, et al. The Alzheimer's Disease Neuroimaging Initiative (ADNI): MRI methods. J Magn Reson Imaging. 2008;27:685-91.

16. Scheltens $P$, Leys $D$, Barkhof $F$, Huglo D, Weinstein HC, Vermersch $P$, et al. Atrophy of medial temporal lobes on MRI in "probable" Alzheimer's disease and normal ageing: diagnostic value and neuropsychological correlates. J Neurol Neurosurg Psychiatry. 1992;55:967-72.

17. Koedam EL, Lehmann M, van der Flier WM, Scheltens P, Pijnenburg YA, Fox $\mathrm{N}$, et al. Visual assessment of posterior atrophy development of a MRI rating scale. Eur Radiol. 2011;21:2618-25.

18. Ferreira D, Cavallin L, Larsson EM, Muehlboeck JS, Mecocci P, Vellas B, et al. Practical cut-offs for visual rating scales of medial temporal, frontal and posterior atrophy in Alzheimer's disease and mild cognitive impairment. J Intern Med. 2015;278:277-90

19. Fischl B, Dale AM. Measuring the thickness of the human cerebral cortex from magnetic resonance images. Proc Natl Acad Sci U S A. 2000;97:11050-5.

20. Fox J, Monette G. Generalized collinearity diagnostics. J Am Stat Assoc. 1992;87:178-83

21. La Joie R, Landeau B, Perrotin A, Bejanin A, Egret S, Pelerin A, et al. Intrinsic connectivity identifies the hippocampus as a main crossroad between Alzheimer's and semantic dementia-targeted networks. Neuron. 2014;81: $1417-28$.

22. Seeley WW, Crawford RK, Zhou J, Miller BL, Greicius MD. Neurodegenerative diseases target large-scale human brain networks. Neuron. 2009;62:42-52.

23. Sestieri C, Shulman GL, Corbetta M. The contribution of the human posterior parietal cortex to episodic memory. Nat Rev Neurosci. 2017;18: 183-92.

24. La Joie R, Perrotin A, Barre L, Hommet C, Mezenge F, Ibazizene M, et al. Region-specific hierarchy between atrophy, hypometabolism, and betaamyloid (Abeta) load in Alzheimer's disease dementia. J Neurosci. 2012;32: 16265-73.

25. Braak H, Braak E. Neuropathological stageing of Alzheimer-related changes. Acta Neuropathol. 1991:82:239-59.

26. Villain N, Fouquet M, Baron JC, Mezenge F, Landeau B, de La Sayette V, et al. Sequential relationships between grey matter and white matter atrophy and brain metabolic abnormalities in early Alzheimer's disease. Brain. 2010;133:3301-14

27. Jacobs HI, Van Boxtel MP, Jolles J, Verhey FR, Uylings HB. Parietal cortex matters in Alzheimer's disease: an overview of structural, functional and metabolic findings. Neurosci Biobehav Rev. 2012;36:297-309.

28. Cabeza R, Ciaramelli E, Olson IR, Moscovitch M. The parietal cortex and episodic memory: an attentional account. Nat Rev Neurosci. 2008;9:613-25.

29. Bai F, Zhang Z, Watson DR, Yu H, Shi Y, Yuan Y, et al. Abnormal functional connectivity of hippocampus during episodic memory retrieval processing network in amnestic mild cognitive impairment. Biol Psychiatry. 2009:65: 951-8.

30. Bokde AL, Karmann M, Born C, Teipel SJ, Omerovic M, Ewers M, et al. Altered brain activation during a verbal working memory task in subjects with amnestic mild cognitive impairment. J Alzheimers Dis. 2010:21:103-18.

31. Spaniol J, Davidson PS, Kim AS, Han H, Moscovitch M, Grady CL. Eventrelated fMRI studies of episodic encoding and retrieval: meta-analyses using activation likelihood estimation. Neuropsychologia. 2009;47:1765-79. 
32. Qi Z, Wu X, Wang Z, Zhang N, Dong H, Yao L, et al. Impairment and compensation coexist in amnestic $\mathrm{MCl}$ default mode network. Neuroimage. 2010;50:48-55

33. Sorg C, Riedl V, Muhlau M, Calhoun VD, Eichele T, Laer L, et al. Selective changes of resting-state networks in individuals at risk for Alzheimer's disease. Proc Natl Acad Sci U S A. 2007;104:18760-5.

34. Ranganath C, Ritchey M. Two cortical systems for memory-guided behaviour. Nat Rev Neurosci. 2012;13:713-26.

35. Moller C, van der Flier WM, Versteeg A, Benedictus MR, Wattjes MP, Koedam EL, et al. Quantitative regional validation of the visual rating scale for posterior cortical atrophy. Eur Radiol. 2014;24:397-404.

36. Lehmann M, Koedam EL, Barnes J, Bartlett JW, Ryan NS, Pijnenburg YA, et al. Posterior cerebral atrophy in the absence of medial temporal lobe atrophy in pathologically-confirmed Alzheimer's disease. Neurobiol Aging. 2012;33: 627 e1-e12.

37. Stern Y. Cognitive reserve. Neuropsychologia. 2009;47:2015-28.

38. Park JY, Na HK, Kim S, Kim H, Kim HJ, Seo SW, et al. Robust identification of Alzheimer's disease subtypes based on cortical atrophy patterns. Sci Rep. 2017;7:43270.

39. Na HK, Kang DR, Kim S, Seo SW, Heilman KM, Noh Y, et al. Malignant progression in parietal-dominant atrophy subtype of Alzheimer's disease occurs independent of onset age. Neurobiol Aging. 2016;47:149-56.

40. Whitwell JL, Shiung MM, Przybelski SA, Weigand SD, Knopman DS, Boeve $B F$, et al. MRI patterns of atrophy associated with progression to $A D$ in amnestic mild cognitive impairment. Neurology. 2008;70:512-20.

41. Vemuri P, Weigand SD, Knopman DS, Kantarci K, Boeve BF, Petersen RC, et al. Time-to-event voxel-based techniques to assess regional atrophy associated with $\mathrm{MCl}$ risk of progression to AD. Neuroimage. 2011:54:985-91.

42. Ten Kate M, Barkhof F, Visser PJ, Teunissen CE, Scheltens P, van der Flier WM, et al. Amyloid-independent atrophy patterns predict time to progression to dementia in mild cognitive impairment. Alzheimers Res Ther. 2017:9:73.

43. Pike KE, Savage G, Villemagne VL, Ng S, Moss SA, Maruff P, et al. Betaamyloid imaging and memory in non-demented individuals: evidence for preclinical Alzheimer's disease. Brain. 2007;130:2837-44.

44. Moon B, Kim S, Park YH, Lim JS, Youn YC, Kim S, et al. Depressive symptoms are associated with progression to dementia in patients with amyloidpositive mild cognitive impairment. J Alzheimers Dis. 2017;58:1255-64.

45. Jansen WJ, Ossenkoppele R, Knol DL, Tijms BM, Scheltens P, Verhey FR, et al. Prevalence of cerebral amyloid pathology in persons without dementia: a meta-analysis. JAMA. 2015;313:1924-38.

46. Liu CC, Liu CC, Kanekiyo T, Xu H, Bu G. Apolipoprotein E and Alzheimer disease: risk, mechanisms and therapy. Nat Rev Neurol. 2013;9:106-18.

47. LaDu MJ, Falduto MT, Manelli AM, Reardon CA, Getz GS, Frail DE. Isoformspecific binding of apolipoprotein E to beta-amyloid. J Biol Chem. 1994;269: 23403-6.

48. Deane R, Sagare A, Hamm K, Parisi M, Lane S, Finn MB, et al. ApoE isoformspecific disruption of amyloid beta peptide clearance from mouse brain. J Clin Invest. 2008;118:4002-13.

49. Hashimoto T, Serrano-Pozo A, Hori Y, Adams KW, Takeda S, Banerji AO, et al. Apolipoprotein E, especially apolipoprotein E4, increases the oligomerization of amyloid beta peptide. J Neurosci. 2012;32:15181-92.

50. van Rossum IA, Vos S, Handels R, Visser PJ. Biomarkers as predictors for conversion from mild cognitive impairment to Alzheimer-type dementia: implications for trial design. J Alzheimers Dis. 2010;20:881-91.

51. Vemuri P, Wiste HJ, Weigand SD, Shaw LM, Trojanowski JQ, Weiner MW, et al. MRI and CSF biomarkers in normal, MCl, and AD subjects: predicting future clinical change. Neurology. 2009;73:294-301.

52. Vemuri P, Wiste HJ, Weigand SD, Knopman DS, Trojanowski JQ, Shaw LM, et al. Serial MRI and CSF biomarkers in normal aging, MCl, and AD. Neurology. 2010;75:143-51.

53. Raz N, Rodrigue KM. Differential aging of the brain: patterns, cognitive correlates and modifiers. Neurosci Biobehav Rev. 2006;30:730-48.

54. Topiwala A, Allan CL, Valkanova V, Zsoldos E, Filippini N, Sexton C, et al. Moderate alcohol consumption as risk factor for adverse brain outcomes and cognitive decline: longitudinal cohort study. BMJ. 2017;357:j2353.

55. Moretti L, Cristofori I, Weaver SM, Chau A, Portelli JN, Grafman J. Cognitive decline in older adults with a history of traumatic brain injury. Lancet Neurol. 2012;11:1103-12.

56. Gorelick PB, Scuteri A, Black SE, Decarli C, Greenberg SM, ladecola C, et al. Vascular contributions to cognitive impairment and dementia: a statement for healthcare professionals from the American Heart Association/American Stroke Association. Stroke. 2011:42:2672-713.

57. Palmqvist S, Zetterberg H, Blennow K, Vestberg S, Andreasson U, Brooks DJ, et al. Accuracy of brain amyloid detection in clinical practice using cerebrospinal fluid beta-amyloid 42: a cross-validation study against amyloid positron emission tomography. JAMA Neurol. 2014;71:1282-9.

58. Landau SM, Lu M, Joshi AD, Pontecorvo M, Mintun MA, Trojanowski JQ, et al. Comparing positron emission tomography imaging and cerebrospinal fluid measurements of beta-amyloid. Ann Neurol. 2013;74:826-36.

\section{Submit your next manuscript to BioMed Central and we will help you at every step:}

- We accept pre-submission inquiries

- Our selector tool helps you to find the most relevant journal

- We provide round the clock customer support

- Convenient online submission

- Thorough peer review

- Inclusion in PubMed and all major indexing services

- Maximum visibility for your research

Submit your manuscript at www.biomedcentral.com/submit
) Biomed Central 